\title{
FOG COMPUTING FOR GEOSPATIAL AND CURRENT GEOSPATIAL STANDARDS
}

DOI: http://dx.doi.org/10.18509/GBP.2017.14

UDC: 528.85:006.3]:[004.75:004.455

\section{Evgeny Panidi}

Saint Petersburg State University, Russia

\begin{abstract}
This position paper formulates the idea of Fog Computing implementation in geospatial domain and underlines some research issues basing on the author's experience in design and implementation of geospatial Web services and geoportals. Main issue covered in the paper is the need of development of the communication interfaces and data models for the geospatial Fog.
\end{abstract}

Keywords: Open Source Standards for Geospatial, Geospatial Fog Computing, Geospatial Web Services, Geospatial Data Interoperability

\section{INTRODUCTION}

Evolution of the Information Technology (IT) impacts on the geospatial domain and leads to movements (tectonic movements sometimes) in the theory and practice of geospatial data management. Good example is the Cloud Computing paradigm that led to the giant improvements in techniques of Spatial Data Infrastructures and formed (in fact) the domain of Web GISs. However, the wheel of IT evolution rolls further and a number of new paradigms seems to be producing some impact onto the Geographic Information Science and Technology. The Fog Computing (FC) is one of such extremely new paradigms, which will be demanded quite possibly in near future.

\section{FOG COMPUTING APPROACH}

FC is relatively new paradigm that was proposed in 2012 [1]. Currently this paradigm is denoted as extension or a next step in evolution of well known Cloud Computing technologies. The essence of the FC in short words can be described as incorporation of the client devices into the Cloud and partial transfer of data storage, processing and exchange activities out of the Cloud core (data center/processing center) to the client devices (Fog nodes), with the save of managing and organizing role of the Cloud core [6], [7].

FC is similar to another one paradigm called Edge Computing that is focused on design and development of client-side (edge) data processing in computer networks. However, in comparison to the Cloud Computing and Edge Computing, the FC can be recognized as a hybrid approach that produce synergy from common use of server-side (in-the-cloud) and client-side computations in distributed computer systems.

FC is based upon the idea of data stale and necessity of data processing in that time and at that place, when and where it is most relevant to do. While some operative data have short period of actuality and demanded locally in most cases, for example on mobile devices. These data have to be processed on mobile devices to exclude lacks of network connection and lag in data transmitting from Cloud core to the mobile device. Some data 
is collected from a number of mobile devices by so-called aggregation nodes of Fog, and is used for comparison and common analysis during medium period. Finally, some data produced by the mobile devices and by the aggregation nodes can be demanded for long period storage at the Cloud core. This approach is relevant in most thematic domains where geospatial data is used. Good example are the meteorological measurements, which are produced intraday on weather stations to compute some medium values locally. Some measurements can be transmitted to the aggregation center where weather forecast for the region is computed for a number of days, and also medium values are transmitted to the state meteorological database for long storage and retrospective analysis.

Fog network can be designed as three-tier (mobile devices/aggregation nodes/Cloud core) as it is described above, or as n-tier [8] depending on demands of application domain. For example, a smart sensors layer can be implemented when monitoring of external conditions is needed. In such a case, FC borders with Internet of Things paradigm that assumes use of smart sensors to provide perception capability of smart devices (smart things).

\section{GEOSPATIAL STANDARDS}

Due to its novelty, the FC is not recognized currently as enabling technology in the geospatial domain. However, the situation is to be changed probably in close time, because of wide range of implementation of the geospatial functionality in many different types of devices and computer systems.

The fundamental issue that prevents FC implementation in geospatial domain is the absence of standards and even best practices for geospatial FC. While the FC itself is an integrating approach that brings together heterogeneous computational resources, the issues of ensuring of the geospatial data and services interoperability in the Fog and between the Fog and traditional Clouds will be the first to be resolved. In this way, most effective approach is the open source standardization that proved its effectiveness already on the example of standards for geospatial Web services developed by Open Geospatial Consortium (OGC) [3], [6], [7], [8].

It is notable also, that OGC works on Sensor Web Enablement (SWE) initiative [4], [5], which incorporates development of a couple of standards for design, implementation and management of the geospatial smart sensors. In fact, these standards are cover partially the needs of Fog architecture, despite the FC is not mentioned. SWE already covers significant number of problems associated with interaction between smart sensors layer and other layers of the Fog.

On the other hand, great number of questions about geospatial Fog architecture are unanswered. While the networking and data exchange between geospatial Cloud core and other nodes of network (aggregation modes, other client nodes, smart sensors) are covered in OGC standards, how the horizontal networking can be organized? Will it be appropriate to extend current standards, or separate standardization will be needed? How to prevent collisions when network configuration appears floating, and almost all nodes appears pluggable?

Similarly, the implementation of client-side (on-the-nodes) geospatial data storage and data processing produce the questions about data structuring and processing software architecture. How to structure and manage data on a number of nodes to ensure scalability? How to monitor and fix the lacks of availability of the data segments stored on unplugged nodes? How to ensure equivalent computations while processing data on different nodes in separate or parallel mode? 
All these questions can be answered only in a result of complex research and development activities of geospatial community. Nevertheless, some case studies conducted with the participation of the author of this paper [2], [11], [12] make it possible to consider the way of extension of the current standards as effective and ensuring backward compatibility with traditional Cloud-based systems. For example, OGC Web Processing Service standard [8] can be extended with additional type of requests to support transmitting of the processing software components onto the client node by demand, and to provide consequent client-side processing of geospatial data.

Design of the basic software (that implement functionality of Cloud services) in a portable manner can also be helpful when deploying Cloud-like services on Fog nodes where computational facilities are limited. In this way, implementation of prototypes of the portable software servers (developed with the participation of author [12]), which implemented OGC Web Coverage Service standard, showed the (limited) possibility of use currently presented Cloud-oriented standards for implementation on-the-nodes data storage and access.

As the promising, the containerization technology can be mentioned also that enables distribution of software on the network in the form of containers (packages where all dependent software components are incorporated and configured). This technology allows to transmit ready-to-use software units through the network (particularly from distributing node onto the processing node on the Fog network).

In addition, the well-known peer-to-peer networking model can be demanded in geospatial Fog. While the geospatial datasets are the big-size datasets in many cases, the storage and computational resources of Fog nodes are assumed to be modest and limited. On the other hand, the aggregation of Fog nodes into groups and building virtual resources on the top of these groups can enable building of highly scalable resources. In this way, peer-to-peer-based file sharing networks can be used potentially as a model approach when designing data structuring techniques for the geospatial Fog.

The list of possible enabling technologies that can be involved when designing open geospatial Fog architecture can be added. Main contemporary issue and the starting point of the geospatial Fog technology development is the proposal of abstract interfaces (standardized and open source) for the communication of geospatial Fog nodes. This interfaces have to be supported by the development of data models, which will enable storage of the geospatial data in distributed and decentralized manner (in contrast to the centralized Cloud-based approach).

\section{CONCLUSIONS}

Fog Computing paradigm was proposed just only in 2012. Currently it appears fast growing and high-potential domain, due to its synergetic nature that consists in aggregation of vertical and horizontal networking in distributed computer systems.

Geospatial Fog Computing remains unstudied currently. However, due to rich perspectives of smart sensors and automated monitoring systems implementation in geospatial missions, Fog Computing architecture tends to be demanded in geospatial domain.

Starting points for research in the geospatial Fog domain are the design and development of open communication interfaces for Fog networks to ensure geospatial data interchange, and development of enhanced data models to ensure distributed and decentralized geospatial data storage. 


\section{REFERENCES}

[1] Bonomi F., Milito R., Zhu J., Addepalli S. Fog Computing and Its Role in the Internet of Things. Proceedings of the first edition of the MCC workshop on Mobile cloud computing, pp. 13-16, 2012. doi:10.1145/2342509.2342512

[2] Kazakov E., Terekhov A., Kapralov E., Panidi E. WPS-based technology for clientside remote sensing data processing. International Archives of the Photogrammetry, Remote Sensing and Spatial Information Sciences, Vol. XL-7/W3, pp. 643-649, 2015. doi:10.5194/isprsarchives-XL-7-W3-643-2015

[3] Open Geospatial Consortium. OpenGIS Web Map Server Interface Implementation Specification. OpenGIS Project Document 00-028, Version 1.0.0, 45 p., 2000.

[4] Open Geospatial Consortium. OGC Sensor Web Enablement Architecture. Best Practice document 06-021r4, Version: 0.4.0, 72 p., 2008.

[5] Open Geospatial Consortium. OGC Sensor Web Enablement: Overview and High Level Architecture. OpenGIS White Paper OGC 07-165, Version: 3, 14 p., 2007.

[6] Open Geospatial Consortium. OGC WCS 2.0 Interface Standard - Core. OGC 09110r3, Version 2.0.0, 2010, 54 p.

[7] Open Geospatial Consortium. Web Feature Service Implementation Specification. OGC 02-058, Version 1.0.0, 105 p., 2002.

[8] Open Geospatial Consortium. OGC WPS 2.0 Interface Standard. OGC Implementation Standard. OGC 14-065, Version 2.0, 133 p., 2015.

[9] OpenFog Consortium Architecture Working Group. OpenFog Architecture Overview. White Paper OPFWP001.0216, 35 p., 2016.

[10] OpenFog Consortium Architecture Working Group. OpenFog Reference Architecture for Fog Computing. OPFRA001.020817, 162 p., 2017.

[11] Panidi E., Kazakov E., Kapralov E., Terekhov A. Hybrid geoprocessing Web services. SGEM2015 Conference Proceedings, Book 2, Vol. 1, pp. 669-676, 2015. doi:10.5593/SGEM2015/B21/S8.084

[12] Panidi E., Terekhov A., Mukhamedzyanov M. Implementation Ways for Web Coverage Service Standard. 2nd Internatonal Scientific Conference GEOBALCANICA 2016 Proceedings, pp. 3-10 2016. doi:10.18509/GBP.2016.01 